\title{
Divergent landscape effects on population connectivity in two co-occurring amphibian species
}

\author{
JONATHAN L. RICHARDSON \\ School of Forestry E Environmental Studies, Yale University, 370 Prospect St., New Haven, CT 06511, USA
}

\begin{abstract}
The physical and environmental attributes of landscapes often shape patterns of population connectivity by influencing dispersal and gene flow. Landscape effects on movement are typically evaluated for single species. However, inferences from multiple species are required for multi-species management strategies increasingly being applied in conservation. In this study, I compared the spatial genetic patterns of two amphibian species across the northeastern United States and estimated the influence of specific landscape features on the observed genetic structure. The spotted salamander (Ambystoma maculatum) and wood frog (Rana sylvatica) share many ecological attributes related to habitat use, phenology and site fidelity. However, I hypothesized that important differences in their movement patterns and life history would create distinct genetic patterns for each species. Using 14 microsatellite loci, I tested for differences in the level of genetic differentiation between the two species across 22 breeding ponds. The effects of eight landscape features were also estimated by evaluating 32 landscape resistance models. Spotted salamanders exhibited significantly higher genetic differentiation than wood frogs. Different landscape features were also identified as potential drivers of the genetic patterns in each species, with little overlap in model support between species. Collectively, these results provide strong evidence that these two amphibian species interact with the landscape in measurably different ways. The distinct genetic patterns observed are consistent with key differences in movement ability and life history between $A$. maculatum and $R$. sylvatica. These results highlight the importance of considering more than one species when assessing the impacts of the landscape matrix on population connectivity, even for ecologically similar species within the same habitats.
\end{abstract}

Keywords: Ambystoma, dispersal, fragmentation, gene flow, landscape genetics, Lithobates sylvaticus, pond-breeding, Rana

Received 12 November 2011; revision received 30 May 2012; accepted 1 June 2012

\section{Introduction}

The landscape separating populations can strongly influence the movement of individuals (dispersal) and genes (gene flow) among populations. Dispersal and gene flow are processes that ultimately influence the demography, evolution and long-term viability of populations, which are all characteristics of great importance to ecologists, evolutionary biologists and conservation scientists. Therefore, understanding how the landscape

Correspondence: Jonathan L. Richardson, Fax: 203-432-3929; E-mail: jonathan.richardson@yale.edu matrix affects movement among populations is of broad interest in biology. Spatial patterns of genetic relatedness across the landscape represent the cumulative gene flow among populations occurring over multiple generations. Methods developed to integrate this spatial genetic data and environmental data provide a framework for evaluating how the landscape matrix is influencing movement and gene flow (Manel et al. 2003; Storfer et al. 2007).

In considering landscape effects on gene flow, most studies evaluate individual species in isolation. However, to apply landscape genetic inferences to conservation management, it is important to know whether results can 
be generalized across species (Schwenk \& Donovan 2011). This requires an approach that directly compares multiple species within the same landscape (Brede \& Beebee 2004; Manier \& Arnold 2006; Steele et al. 2009; Goldberg \& Waits 2010a; Delaney et al. 2010; Olsen et al. 2011). Landscape features can have varied impacts on the movement of species, and understanding the similarities and differences among species is a key focus of multispecies conservation planning (Nicholson \& Possingham 2006; Schwenk \& Donovan 2011).

In the present study, I compared the patterns of population genetic structure of two pond-breeding amphibian species across the northeastern United States. The spotted salamander (Ambystoma maculatum) and wood frog $($ Rana sylvatica $=$ Lithobates sylvaticus $)$ are widely distributed across northeastern North America, both having recolonized this region from southern refugia following the last glacial maximum ( 20 kya; Zamudio \& Savage 2003; Lee-Yaw et al. 2008). Both species use similar aquatic and terrestrial habitats, with breeding limited primarily to the same temporary pond habitats. Additionally, A. maculatum and R. sylvatica experience explosive breeding bouts during the same period in late winter, followed by metamorphosis and migration away from the ponds within a few months. This biphasic life cycle ensures that they interact with both freshwater wetlands and terrestrial uplands on the landscape. Furthermore, both species exhibit high site fidelity (philopatry), which promotes insularity of populations (Berven \& Grudzien 1990; Vasconcelos \& Calhoun 2004; Boone et al. 2006; Meier 2007).

Despite their many similarities, there are several important differences between spotted salamanders and wood frogs that may affect the spatial genetic patterns of each species. Contrasting modes of locomotion may contribute to the disparate estimates of juvenile and adult dispersal for each species (mean movement distance away from breeding pond: A. maculatum $=118 \mathrm{~m}$, $R$. sylvatica $=193 \mathrm{~m}$; maximum distance observed: A. maculatum $=467 \mathrm{~m}, R$. sylvatica $=2530 \mathrm{~m}$; Berven \& Grudzien 1990; Montieth \& Paton 2006; Semlitsch \& Skelly 2008). This disparity in vagility appears to apply more broadly among other species of frogs and salamanders (Smith \& Green 2005). Additionally, spotted salamanders are longer lived, with a mean age of 7 years compared to 2.5 years for wood frogs (Flageole \& Leclair 1992; Sagor et al. 1998). This leads to increased iteroparity and overlapping generations within $A$. maculatum, attributes that can slow genetic divergence among populations (Nunney 1993) and lead to a longer lag time in detecting the impact of new landscape features (Anderson et al. 2010).

Previous research on these two and related species provides evidence that several landscape features can shape genetic patterns. While not consistent among studies, there is support from A. maculatum and $R$. sylvatica that distance, slope, land cover, rivers and open water are associated with decreased genetic connectivity (Lee-Yaw et al. 2009; Zellmer \& Knowles 2009; Greenwald et al. 2009). Evidence from other pondbreeding amphibians suggests that roads (Lesbarrères et al. 2006) and wetland density (Scribner et al. 2001) can also influence genetic patterns across a landscape. A study from the western United States examining two species closely related to A. maculatum and $R$. sylvatica found that different land cover classes were associated with genetic connectivity in each species, possibly corresponding to different soil moisture tolerances (Goldberg \& Waits 2010a). On the basis of this previous research and the ecology of the focal species, I predicted that slope, land cover, wetland density and the presence of rivers and roads on the landscape might influence the genetic connectivity of $A$. maculatum and $R$. sylvatica populations throughout the study region.

In this study, my objectives were to (i) test whether co-occurring populations of spotted salamanders and wood frogs have different spatial genetic patterns across the landscape and (ii) identify specific features of the landscape matrix that influence the observed genetic patterns. I hypothesized that differences in movement behaviour would create distinct spatial genetic patterns for each species. Specifically, the less vagile spotted salamander was predicted to exhibit greater genetic divergence and structure among populations than the wood frog. Similar patterns between species would suggest that they are influenced by the landscape in comparable ways, as would be predicted from their ecological similarities. Alternatively, overlapping generations and iteroparity within the salamanders could slow genetic differentiation relative to wood frogs, attenuating genetic structure resulting from the landscape. If these life history differences drive genetic patterns in this system, landscape features of contrasting ages (old vs. new) would be expected to highlight the differences between species. In this case, the longer-lived salamanders would be expected to exhibit weaker genetic structuring in response to modern elements (e.g. roads) than long-standing features (e.g. topography) relative to wood frogs. The ecological and life history attributes of $A$. maculatum and $R$. sylvatica allow me to assess how the similarities and differences between species can influence population genetic patterns across space.

\section{Methods}

\section{Study region and sampling design}

The study region (Fig. 1) was selected because it encompasses a heterogeneous landscape matrix suit- 


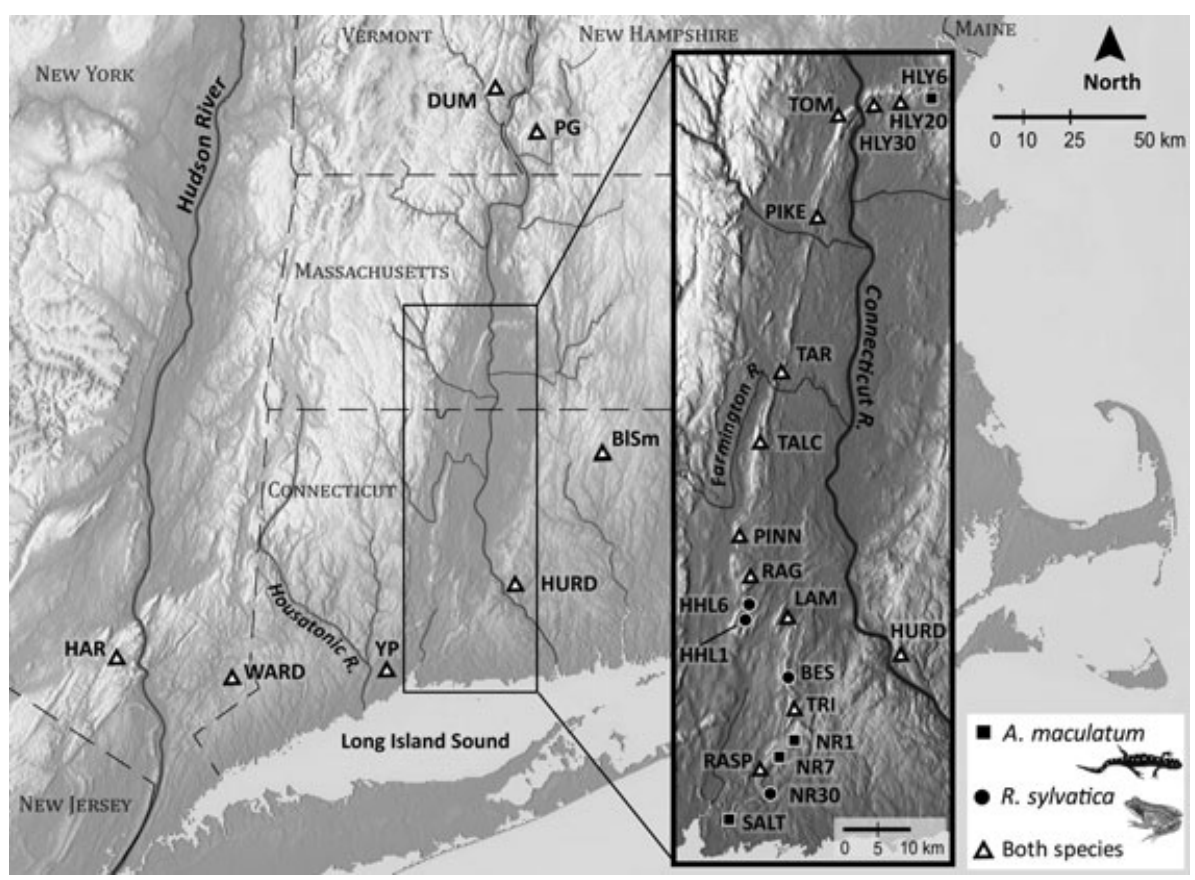

Fig. 1 Relief map of the study region and sampling sites (lighter coloring represents higher elevations). Symbols denote which species were collected at each pond. Much of the sampling effort was concentrated along the Metacomet Ridge, the thin high elevation feature stretching between the SALT and HLY6 sites.

able for evaluating the contribution of numerous landscape features to patterns of gene flow. The area is dominated by broadleaf deciduous and mixed forests and today is intermixed with developed and agricultural lands. There are also many linear features present of natural and anthropogenic origin that may serve as impediments to gene flow (e.g. roads and rivers). Sampling was concentrated along the Metacomet Ridge, a basalt ridge of modest topographic relief (100-300 $\mathrm{m}$ above surrounding lowlands) that runs through the lower Connecticut River Valley. The impervious bedrock creates a high density of temporary pond habitats, while the forested landscape covering this ridge is in sharp contrast to the surrounding valley characterized by urban development and agriculture (Lee 1985).

Samples were collected during the springs of 20082010 from 26 ponds with breeding populations of A. maculatum and $R$. sylvatica, including 18 ponds where both species were sampled (Fig. 1, Table S1, Supporting information). For salamanders, a single egg was removed from each egg mass present within a pond (up to 40 per pond), and eggs were reared to hatching in the laboratory. Female spotted salamanders often deposit a large primary mass and a smaller satellite mass (Petranka 1998). Eggs were not collected from satellite masses to avoid duplicate sampling within sibships. For wood frogs, I also collected a single egg from each mass when possible. However, wood frogs have a much shorter embryonic stage before hatching. In some ponds, I collected early-stage tadpoles by dipnetting randomly throughout the pond after hatchlings had dispersed from the communal egg mass deposition area. Only one tadpole was collected per dip to avoid sampling from spatially clustered kin groups (Halverson et al. 2006). The inclusion of numerous full-sibling pairs may bias estimates of population genetic parameters (Goldberg \& Waits 2010b). However, an analysis in the program COLONY (Wang 2004) estimated that full siblings represented $<1 \%$ of the data set for both species (proportion of data set identified as full siblings: A. maculatum $=0.006, R$. sylvati$c a=0.003)$.

\section{Microsatellite amplification and screening}

Genomic DNA was extracted from the tail fin tissue of each sample using the Qiagen tissue extraction kit. DNA was used in polymerase chain reactions (PCR) that differed for each primer set (Appendix S1, Supporting information). For salamanders, I amplified 10 tetra- and four dinucleotide repeat microsatellite loci. Wood frog samples were amplified at 10 tetra- and five dinucleotide loci. PCR products were identified using capillary eletrophoresis on an ABI 3730 DNA Analyzer. GenEMAPPER v3.7 was used to score alleles 
and Microsatellite tOolKit v3.1 (Park 2001) was used to check for scoring errors.

Independence of loci was verified by testing for linkage disequilibrium in FSTAT v2.9.3 (Goudet 1995). Deviations from Hardy-Weinberg equilibrium (HWE) for each locus were also estimated, with significance assessed using 10000 randomizations. Null allele frequencies were obtained using MICRO-CHECKER v2.2.3 (Van Oosterhout et al. 2004). Loci exhibiting null allele frequencies $>0.15$ were excluded from later analyses (Chapuis \& Estoup 2007). Hereafter, I will refer to individuals sampled from the same pond (i.e. breeding aggregation) as populations.

\section{Population genetic structure}

Summary statistics and genetic differentiation. Several indices of genetic diversity were calculated using FSTAT, including heterozygosity, allelic richness and the inbreeding coefficient $\left(F_{\mathrm{IS}}\right)$. Genetic differentiation was estimated using $F_{\mathrm{ST}}$ (Weir \& Cockerham 1984). $F_{\mathrm{ST}}$ across all populations and pairwise $F_{\mathrm{ST}}$ values between populations were calculated, and values significantly $>0$ (zero representing panmixia among populations) were identified using bootstrap resampling (10 000 randomizations). Bonferroni corrections were applied to estimates of pairwise differentiation to assess significance at an adjusted alpha. There are several metrics of differentiation that have found support in the literature recently (Hedrick 2005; Jost 2008; Meirmans \& Hedrick 2011). Here, $F_{\text {ST }}$ was used because the associated evolutionary interpretation is clearer than for other metrics (Whitlock 2011).

Estimates of genetic differentiation can be influenced by the genetic variation present in the sample (Hedrick 2005), whereby the maximum attainable levels of differentiation decrease as heterozygosity increases. To account for this, I calculated a standardized $F_{\mathrm{ST}}\left(F_{\mathrm{ST}^{\prime}}\right)$ for each species following the methods of Meirmans (2006) in the program RECODE. Standardized $F_{\mathrm{ST}}$ values were obtained by dividing the original estimates of $F_{\mathrm{ST}}$ by the maximum $F_{\mathrm{ST}}$ values possible for each data set (Hedrick 2005). Because $F_{\mathrm{ST}}$ and $F_{\mathrm{ST}}$ ' have different properties, I use both in this study. $F_{\mathrm{ST}}$ is necessary for comparisons between species or markers, while $F_{\mathrm{ST}}$ is appropriate for single-species comparisons among studies using the same markers and loci (Meirmans 2006; Chan \& Zamudio 2009). However, heterozygosity levels for studies of the same species should still be considered, as large differences may prevent an accurate comparison of genetic differentiation using standard $F_{\mathrm{ST}}$.

To test whether levels of differentiation differ significantly between spotted salamanders and wood frogs, I used two methods to compare the estimates of $F_{\mathrm{ST}}$ and $F_{\mathrm{ST}}{ }^{\prime}$ in each species. First, I conducted permutation tests on $F_{\mathrm{ST}}$ values in $\mathrm{R}$ v2.13 ( $\mathrm{R}$ Development Core Team 2011; exactRankTests package: Hothorn \& Hornik 2006). The nonparametric permutation test creates a null distribution of $F_{\mathrm{ST}}$ using data from both species to test for a significant difference in the observed $F_{\mathrm{ST}}$ distributions between species. Second, I conducted bootstrap resampling (10 000 resamples) of the pairwise $F_{\mathrm{ST}}$ values to determine whether the $95 \%$ confidence intervals for each species overlapped. This analysis was conducted with the boot package in $\mathrm{R}$ (Canty \& Ripley 2011).

Effective population size $\left(N_{\mathrm{e}}\right)$ can also have a strong influence on genetic patterns, particularly the rate of loss of rare alleles and heterozygosity via drift (Allendorf \& Luikart 2007). A large $N_{\mathrm{e}}$ acts as a reservoir of genetic variation, reducing the effects of drift and slowing genetic divergence (Gauffre et al. 2008). To account for any inherent differences in $N_{\mathrm{e}}$ between species, I estimated this parameter for each population using the moment-based linkage disequilibrium method implemented in LDNE, with the random mating model and a critical value of 0.01 (Waples \& Do 2008). For comparisons, I calculated the harmonic mean of $N_{\mathrm{e}}$ for each species using only the 18 ponds from which both species had been collected. The harmonic mean is the most appropriate measure of central tendency in $N_{\mathrm{e}}$ because the consequences of genetic drift scale with $1 / N_{e}$, and $N_{\mathrm{e}}$ estimates are rarely normally distributed (Crow \& Kimura 1970; R. Waples, pers. comm.).

Isolation by distance. Gene flow can be strongly correlated with the geographic distance separating populations (Jenkins et al. 2010). This pattern of isolation by distance (IBD) creates a clinal distribution of genetic similarities across the landscape. I tested for IBD by performing Mantel tests of matrix correlation with 10000 permutations in $\mathrm{R}$. Mantel tests account for the nonindependence of pairwise matrix elements using permutations to assess the significance of correlations. There has been some debate regarding the efficacy of the Mantel test (Raufaste \& Rousset 2001; Castellano \& Balletto 2002; Balkenhol et al. 2009). Much of the criticism relates to reduced statistical power (type II error) rather than spurious insights, and Mantel tests remain an appropriate method for data consisting entirely of distance measures (Legendre \& Fortin 2010).

Bayesian estimates of genetic structure. One goal of this study was to characterize and compare the genetic structure of these two species across the study region. To do this, I used a Bayesian clustering method implemented in StRUCTURE v2.3.3 (Pritchard et al. 2000). This 
approach infers distinct genetic groups by estimating $\operatorname{Pr}(X \mid K)$, the probability of the observed genetic data given $K$ number of genetic clusters. STRUCTURE delineates these clusters by grouping individuals in a manner that minimizes deviations from Hardy-Weinberg and linkage equilibria within groups. Plotting each individual's membership probability (to each cluster) provides a visual representation of genetic structure. Estimating $K$ can be difficult, especially at large spatial scales where IBD is driving genetic patterns (Guillot et al. 2009). In the present study, I was more interested in comparing the general patterns of genetic clustering between the two species rather than estimating a definitive number of genetic groups.

I ran 10 independent simulations at each value of $K$ between 1 and 10, as exploratory analyses supported $K<10$. Each run consisted of 750000 iterations following a 250000 burn-in period. I included priors allowing admixture and correlated allele frequencies among groups, as well as a sampling location prior that can increase the resolution of data with less pronounced structure without creating artificial clusters (Hubisz et al. 2009). Appropriate $K$ values were selected using the natural $\log$ of $\operatorname{Pr}(X \mid K)$ values reported by STRUCTURE and the $\Delta K$ criterion (Evanno et al. 2005). Replicate runs were averaged using CLUMPP v1.1 (Jakobsson \& Rosenberg 2007) and plotted using DISTRUCT v1.1 (Rosenberg 2004).

\section{Landscape influence on genetic structure}

To estimate the influence of specific landscape features on the observed genetic patterns, I tested a series of 32 landscape resistance models for each species. These models use resistance surfaces to represent the relative permeability of the landscape and are commonly used to model landscape effects on gene flow (Spear et al. 2010). This analysis was performed in two steps. First, cost values for eight landscape features were selected based on their correlation with genetic distance among populations. Second, these cost values were incorporated into landscape models that were used to identify which landscape features most strongly affected genetic patterns using matrix correlation and Akaike Information Criterion (AIC) model selection methods. To ensure that model inferences were robust, landscape models were tested using $F_{\mathrm{ST}}$ and $D_{\mathrm{ps}}$, a second genetic distance measure based on the proportion of shared alleles (Bowcock et al. 1994). All statistical analyses were conducted in $\mathrm{R}$ v2.13.

Model development and landscape data acquisition. Landscape resistance models were run using CIRCUITSCAPE v3.5 with data rasters created in ARCMAP v9.3 (ESRI). CIRCUITSCAPE uses electrical circuit theory as a framework for predict- ing movement and gene flow across a landscape (McRae et al. 2008). Landscapes represent conductive surfaces where habitats of low suitability are assigned high costs and impart higher resistance to the simulated current. By analogy, these high cost areas should hinder dispersal and gene flow. A circuit-based approach can evaluate the contributions of multiple dispersal routes, thereby relaxing assumptions inherent to other methods that require dispersing individuals to choose a single optimal path (McRae et al. 2008; Sawyer et al. 2011). I ran CIRCUITSCAPE using the 'pairwise' mode and calculated resistance among ponds specifying a four-neighbour cell connection scheme.

The following landscape features were assessed: land cover, slope, wetland habitat, rivers, roads and rail lines. Most data were obtained at $30 \times 30 \mathrm{~m}$ resolution and resampled to $60 \times 60 \mathrm{~m}$ for computational efficiency. Land cover data from the National Land Cover Database (USGS) were consolidated into three classes based on the similarity of the land cover types to reduce the number of models to a number that I could feasibly test (Table S4, Supporting information). Saltwater, estuaries, and brackish water were masked out of all landscape models.

Slope values were calculated from a digital elevation model, while wetland data were obtained from the National Wetlands Inventory (USFWS). As both species generally avoid large, permanent water bodies (Cunningham et al. 2007; J. L. Richardson, pers. observ.), analyses only included wetlands smaller than 5 acres (Appendix S2, Supporting information). GIS data for rivers were obtained from the National Hydrography Dataset (USGS). Rivers were divided into two classes using the Strahler numbering method. Rivers of stream order 6 and 7 were merged into one class (hereafter 'Large Rivers') and 4th and 5th order rivers into a second class ('Medium Rivers'), while lower order streams were excluded. Road features were similarly divided into groups: a 'Divided Highways' feature included all limited-access and divided highways, while a 'Major Roads' class included major, nondivided state and national highways. Other roads were excluded from analyses. I also included railroad tracks as another linear feature.

\section{Landscape resistance modelling:}

Step 1 - Selection of cost values. When using resistance surfaces, cost values assigned to the various landscape elements can be influential in the inferences provided by landscape models (Rayfield et al. 2010; Spear et al. 2010). To parameterize resistance surfaces, most studies rely on expert opinion that can be subjective or even conflicting among experts (Spear et al. 2010). Here, I selected costs by assessing a range of resistance values 
for each feature and determining statistically which had the best fit to the genetic data (Pérez-Espona et al. 2008). Partial Mantel tests (with 10000 permutations) were used to estimate the correlation between the genetic distance and the resistance distance (from CIRCUITSCAPE) between each pond, controlling for the effects of geographic distance. The objective of this analysis was not to obtain definitive cost values for these species in all contexts, but rather to select reasonable costs for this region that were supported by the data.

Five to eight cost values were tested for each landscape feature (Table S5, Supporting information), with all nonfeature cells assigned a cost of one. I selected the cost that maximized the partial correlation coefficient. In a few cases, coefficients increased asymptotically, in which case the lowest cost value that approached the asymptote was selected. With wetlands, I tested values between 0.0001 and 10 to consider the possibility that these breeding habitats could serve as sources of gene flow. CIRCUITSCAPE is not able to produce meaningful results with negative cost values, which may be the most appropriate way to model breeding habitats. See Appendix S2 (Supporting information) for extended details on cost estimation.

Step 2 - Additive landscape models. The cost values obtained in Step 1 were incorporated into a series of additive landscape resistance models. These models tested the relative contribution of each feature by starting with single-feature models and adding other landscape elements to create more complex models. To generate a reasonable number of models to test, I consolidated both river classes into a single feature and merged both road classes and railroads into a single roads feature, with each feature category retaining its cost value obtained in Step 1.

Two methods were used to assess the support of each model. First, I used partial correlation coefficients as a metric of model fit to the genetic data. Standard and partial Mantel tests were performed to estimate significance of the fit with and without the influence of geographic distance between ponds. Second, I used model selection methods based on the corrected AIC ( $\mathrm{AIC}_{c}$ ) to assess which models were closer to the 'true' model taking parsimony into account (Burnham \& Anderson 2002). I calculated the difference in $\mathrm{AIC}_{c}\left(\Delta \mathrm{AIC}_{c}\right)$ between the minimum $\mathrm{AIC}_{c}$ model and all other models. Appendix S2 (Supporting information) contains expanded methods for this resistance modelling analysis.

AIC methods assume independence among data points (i.e. normally distributed error terms; Burnham \& Anderson 2002). I assessed the level of nonindependence for the genetic data at each pond using methods developed by Goldberg \& Waits (2010a) and detailed in Appendix S3 (Supporting information). Briefly, I estimated the degree of autocorrelation among the residuals of the regression between genetic and geographic distance for each sampled pond. I compared the empirical data obtained for each pond with a null distribution created by bootstrap resampling the full data set 10000 times. Significance was assessed after correcting for multiple tests using Holm's procedure, a less conservative correction than standard Bonferroni (Holm 1979).

\section{Results}

\section{Genotype screening}

There was no evidence of linked loci for either species after applying Bonferroni corrections. Across both species, five of the 29 loci exhibited some level of departure from HWE; however, these deviations were limited to a small number of populations in four of these five loci. One wood frog locus (D55) was out of HWE in nearly half of the study populations and was the only locus that exceeded the threshold null allele frequency $(v=0.182)$. Therefore, locus D55 was removed from the data set and subsequent analyses included 22 populations and 14 loci for each species. In total, 626 salamander $($ mean $=28.5$ per pond $)$ and 469 wood frog (21.3 per pond) individuals were included in analyses (Table S1, Supporting information).

\section{Summary statistics and genetic differentiation}

Heterozygosity, mean $F_{\mathrm{IS}}$ and allelic richness were all slightly higher for wood frogs than spotted salamanders (Table S1, Supporting information), and significant differentiation was detected across populations for each species $(P<0.001)$. Both permutation tests and bootstrap resampling indicated that $F_{\mathrm{ST}}$ values were significantly different between the two species $(P<0.01)$. Specifically, spotted salamanders exhibited more than twice the level of differentiation as wood frogs (A. maculatum mean $F_{\mathrm{ST}}=0.033 ; R$. sylvatica $F_{\mathrm{ST}}=0.016$; Tables S2 and S3, Supporting information). Standardized $F_{\mathrm{ST}}\left(F_{\mathrm{ST}^{\prime}}\right)$ estimates produced similar results, with significantly higher values for $A$. maculatum and nonoverlapping $95 \%$ confidence intervals (A. maculatum $F_{\mathrm{ST}^{\prime}}=0.110 ; 95 \% \mathrm{CI}$, $0.104-0.115 ;$ R. sylvatica $F_{\mathrm{ST}^{\prime}}=0.091 ; 95 \%$ CI, $0.085-$ 0.096). Collectively, these data support the prediction of higher levels of differentiation among salamander populations than wood frogs. Estimates of effective population size were large and varied widely among populations for both species (Table S1, Supporting information). Estimates were larger for A. maculatum; 


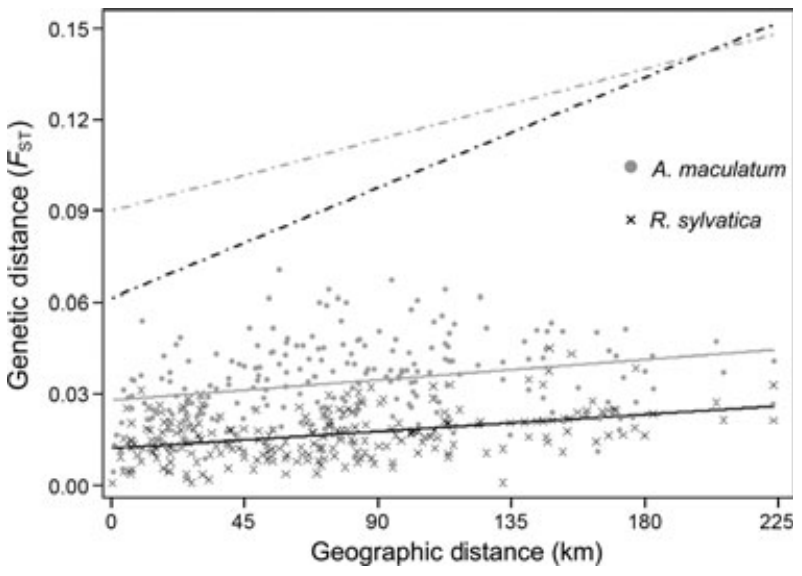

Fig. 2 Regression of pairwise genetic and geographic distance between populations. Both spotted salamanders (gray lines) and wood frogs (black lines) exhibited a significant correlation between genetic and geographic distance, indicating a pattern of isolation by distance using both $F_{\mathrm{ST}}$ (solid lines - R. sylvatica: $r=0.397, P=0.001 ;$ A. mbystoma: $r=0.284, P=0.009$ ) and standardized $F_{\mathrm{ST}^{\prime}}$ (dashed lines - R. sylvatica: $r=0.481, P<0.001$; A. maculatum: $r=0.308, P=0.006)$.

however, the difference was not significant. The mean $N_{\mathrm{e}}$ for $A$. maculatum was 570 and 456 for $R$. sylvatica.

\section{Isolation by distance}

There was a strong pattern of isolation by distance for both species (Fig. 2), with the wood frog exhibiting stronger IBD than the spotted salamander using either $F_{\mathrm{ST}}$ (R. sylvatica: $r=0.397, \quad P=0.001 ; \quad$ A. maculatum: $r=0.284, P=0.009)$ or $F_{\mathrm{ST}^{\prime}} \quad(R$. sylvatica: $r=0.481$, $P<0.001 ;$ A. maculatum: $r=0.308, P=0.006)$.

\section{Bayesian estimates of structure}

For spotted salamanders, the value of $K$ increased asymptotically between 1 and $10 . \Delta K$ values, representing the rate of change along this asymptote, supported both $K=2$ and $K=5$. Using either $K, A$. maculatum exhibit a clear genetic cluster at the northern terminus of the Metacomet Ridge, incorporating populations HLY30, HLY20, HLY6 and, to a lesser degree, TOM and DUM (Fig. 3A,B). When $K=5$, A. maculatum exhibit two more geographically distinct clusters: the New York populations (HAR and WARD) and two Massachusetts populations (PIKE and TOM). The other two clusters were more spatially admixed (Fig. 3A,B). For wood frogs, $\operatorname{Pr}(\mathrm{X} \mid K)$ values peaked at $K=3$, supporting three genetic groups. One cluster dominated the northern half of the study region and generally overlapped with the most distinct A. maculatum cluster, but expanded to include three more populations (PIKE, TOM and PG; Fig. 3C). A second genetic group was ubiquitous in the southern part of the region, but also appeared in several midlatitude populations. The third supported cluster was restricted to the populations central within the study area (LAM, HHL1, HHL6, RAG).

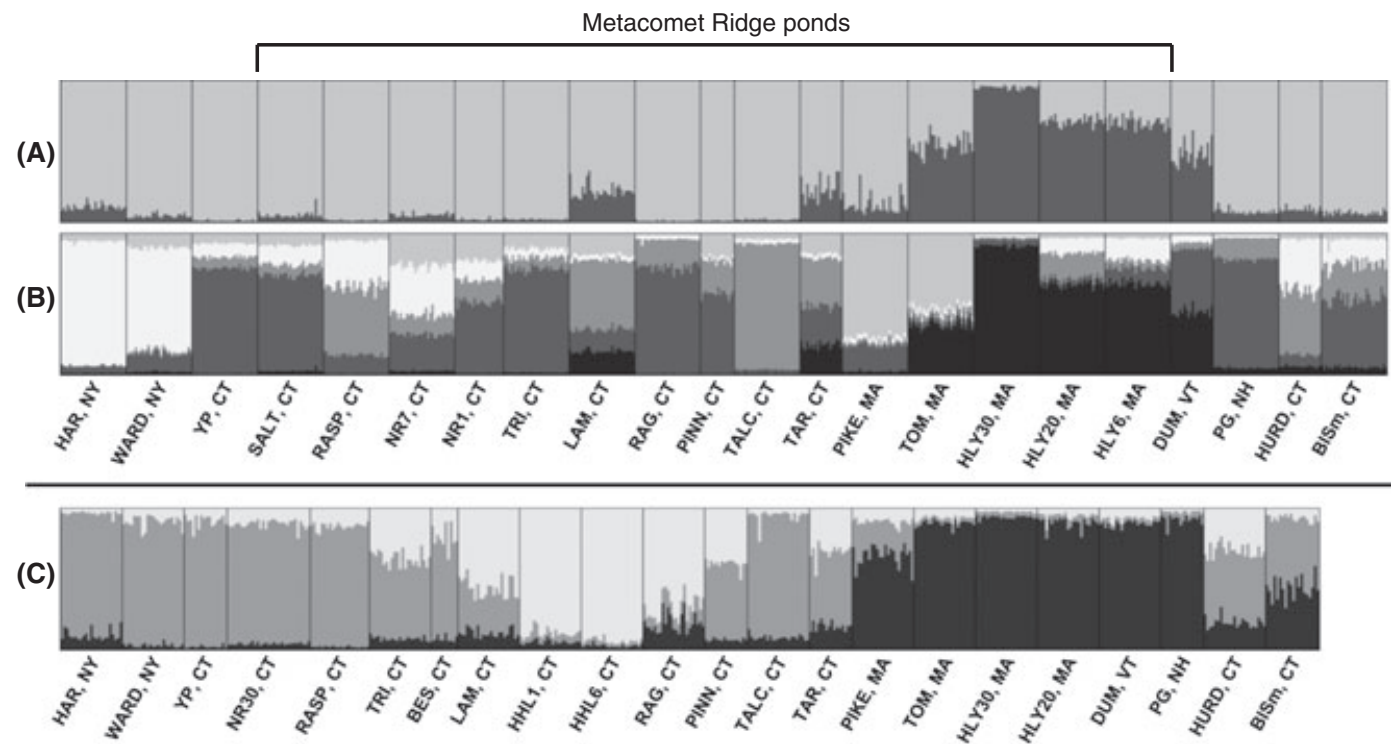

Fig. 3 Bayesian clustering results from STRUCTURE. Sampling sites are delimited by vertical black bars with the pond name and state identified below. Each bar within a pond represents a single individual, and the colors of each bar represent the proportion of that individual assigned to the $K$ genetic clusters. Results support two genetic cluster values for spotted salamanders: $K=2$ (A) and $K=5$ (B). The most distinct group encompassed the ponds at the northern terminus of the Metacomet Ridge. For wood frogs, three genetic clusters were clearly supported by the genetic data $(K=3)$, roughly divided into southern, central and northern clusters $(C)$. 
Table 1 Cost values used in the additive landscape resistance models. A range of cost values was tested for each landscape feature, and the value with the strongest association with the genetic data was selected (Table S5, Supporting information). The original 14 land cover (LC) categories were consolidated into three classes to reduce the number of models to test (see Table S4, Supporting information for details). For wood frogs, several landscape features did not have significant $P$-values for the range of costs tested (numbers in italics)

\begin{tabular}{lll}
\hline Landscape Feature & $\begin{array}{l}\text { Ambystoma } \\
\text { maculatum }\end{array}$ & $\begin{array}{l}\text { Rana } \\
\text { sylvatica }\end{array}$ \\
\hline Divided Highways & 100 & 1000 \\
Major Roads & 25 & 10 \\
Large Rivers & 500 & 10 \\
Medium Rivers & 150 & 10 \\
Railroads & 100 & 200 \\
Wetlands (<5acres) & 0.5 & 0.1 \\
Slope & degrees +50 & degrees +1 \\
Land Cover class A & 1 & 1 \\
$\quad$ (all forest types) & & 3 \\
LC class B (agriculture & 3 & 15 \\
$\quad$ \& open space) & & \\
LC class C (developed & 10 & \\
$\quad$ \& open water) & & \\
\hline
\end{tabular}

\section{Landscape influence on genetic structure}

In resistance model parameterization, supported cost values differed between the two species (Table 1; Table S5, Supporting information). Divided highways supported a much higher cost for wood frogs than for salamanders, whereas other major roads did not warrant high costs. In contrast, very low costs of rivers were supported for wood frogs, while high values were supported for salamanders. Wetland costs were well below one for wood frogs, consistent with the biological interpretation of wetlands as breeding habitat serving to facilitate gene flow. The higher cost associated with slope suggests that topography may have a stronger influence on salamanders than wood frogs (Table 1). Land cover costs were similar for both species; however, developed areas and open freshwater obtained a higher cost for wood frogs (Table 1). It is important to note that several landscape feature costs in the wood frog analysis were not strongly associated with the genetic data, but still showed a range of correlation strengths (Table S5, Supporting information). While these nonsignificant feature costs probably have minimal influence on some wood frog models, including them allows for a direct comparison of each model for each species.

On the basis of the significance of the partial Mantel tests, 23 of the 32 additive landscape models tested were supported for spotted salamanders using both $F_{\mathrm{ST}}$ and $D_{\mathrm{ps}}$ (Table 2; Table S6, Supporting information).
$\mathrm{AIC}_{c}$ scores indicate similar model rankings (Table 2). Considering either criteria, 12 of the top 13 models for A. maculatum all included the rivers feature, with isolation by distance (IBD) ranked fifth. In general, fewer models were associated with the genetic data for wood frogs, with IBD the most strongly supported (Table 2; Table S6, Supporting information). The top nine models (following IBD) using $F_{\mathrm{ST}}$ all incorporated roads. Using $D_{\mathrm{ps}}$ still results in the top six wood frog models (after IBD) incorporating roads, but the model rankings differ, with decreased significance (Table 2; TableS6, Supporting information). However, the top two models (IBD and roads) are the same using $F_{\mathrm{ST}}$ and $D_{\mathrm{ps}}$. Results are concordant between $F_{\mathrm{ST}}$ and $D_{\mathrm{ps}}$ in wood frogs using partial Mantel tests; however, there is much greater variation in $\Delta \mathrm{AIC}_{c}$ values using $D_{\mathrm{ps}}$, with the top ranking models incorporating wetland density (Table S6, Supporting information). Tests of independence indicate that there was no significant evidence of autocorrelation associated with any pond, confirming the suitability of AIC for this study. Lastly, standard Mantel tests indicate that geographic distance alone accounts for some of the variation in genetic distance among ponds (Table S6, Supporting information). However, controlling for the effect of geographic distance using the partial Mantel test highlights the significant influence of other landscape features tested.

\section{Discussion}

The results of this study indicate that genetic structure in two co-occurring amphibian species differs in ways that can be predicted by their movement patterns and life history attributes. Genetic differentiation among A. maculatum populations was significantly greater than in $R$. sylvatica. The two species also differed in the spatial pattern of genetic structuring across the study landscape. Additionally, landscape resistance models identified different landscape features as strongly shaping the genetic patterns for each species. These results suggest that wood frog populations across this region experience higher genetic connectivity than spotted salamanders, which is consistent with the hypothesis that movement ability is important in shaping spatial genetic patterns. Concordant results from the three analytical approaches (genetic distance, landscape resistance modelling and clustering) also indicate that the differences in landscape response observed between species are substantial.

\section{Ecological and life history drivers}

Strong evidence exists from mark-recapture and tracking studies that average movement distances are higher 
Table 2 Landscape resistance models assessed using partial correlation coefficients ( $r$, controlling for distance between ponds) and $\mathrm{AIC}_{c}$ scores for $F_{\mathrm{ST}}$ and $D_{\mathrm{ps}}$ genetic distance measures. Only the ten best-supported models are presented here (see Table S6, Supporting information for the full set of models). Models incorporated five landscape features: Roads (Rd), Rivers (Riv), Wetlands (W), Slope (S) and Land Cover (LC). Output includes the $P$-value from partial Mantel tests $(P)$ and the difference in AIC $_{c}\left(\Delta \mathrm{AIC}_{c}\right)$ between the best model (i.e. minimum $\mathrm{AIC}_{c}$ ) and all other models. Landscape models are ranked by the $F_{\mathrm{ST}}$ correlation coefficient to accommodate the isolation by distance model (IBD), whose $\mathrm{AIC}_{c}$ is obtained from simple linear regression and is not directly comparable to the other $\mathrm{AIC}_{c}$ values

\begin{tabular}{|c|c|c|c|c|c|c|}
\hline \multirow[b]{2}{*}{ Landscape Model } & \multicolumn{3}{|l|}{$F_{\mathrm{ST}}$} & \multicolumn{3}{|l|}{$D_{\mathrm{ps}}$} \\
\hline & $r$ & $P$ value & $\Delta \mathrm{AIC} c$ & $r$ & $P$ value & $\Delta \mathrm{AIC} c$ \\
\hline \multicolumn{7}{|c|}{ Ambystoma maculatum } \\
\hline Riv & 0.332 & 0.003 & 0.0 & 0.318 & 0.007 & 0.0 \\
\hline $\operatorname{Riv}+\mathrm{Rd}$ & 0.323 & 0.003 & 1.5 & 0.313 & 0.005 & 0.8 \\
\hline W+Riv & 0.300 & 0.010 & 5.1 & 0.284 & 0.017 & 5.2 \\
\hline $\mathrm{W}+\mathrm{Riv}+\mathrm{Rd}$ & 0.299 & 0.006 & 5.3 & 0.273 & 0.016 & 6.8 \\
\hline IBD & 0.284 & 0.008 & - & 0.306 & 0.006 & - \\
\hline $\mathrm{S}+\mathrm{LC}+\mathrm{W}+\mathrm{Riv}+\mathrm{Rd}$ & 0.267 & 0.003 & 9.9 & 0.242 & 0.008 & 10.6 \\
\hline $\mathrm{S}+\mathrm{Riv}+\mathrm{Rd}$ & 0.265 & 0.001 & 10.2 & 0.255 & 0.003 & 9.0 \\
\hline $\mathrm{S}+\mathrm{LC}+\mathrm{Riv}+\mathrm{Rd}$ & 0.263 & 0.004 & 10.4 & 0.241 & 0.010 & 10.8 \\
\hline S+LC+Riv & 0.258 & 0.005 & 11.0 & 0.238 & 0.009 & 11.2 \\
\hline $\mathrm{S}+\mathrm{LC}+\mathrm{W}+\mathrm{Riv}$ & 0.258 & 0.003 & 11.0 & 0.239 & 0.009 & 11.0 \\
\hline \multicolumn{7}{|l|}{ Rana sylvatica } \\
\hline IBD & 0.397 & 0.002 & - & 0.458 & 0.001 & - \\
\hline $\mathrm{Rd}$ & 0.300 & 0.008 & 0.0 & 0.329 & 0.015 & 813.1 \\
\hline $\mathrm{S}+\mathrm{W}+\mathrm{Rd}$ & 0.295 & 0.026 & 0.7 & 0.133 & 0.213 & 1296.7 \\
\hline $\mathrm{S}+\mathrm{LC}+\mathrm{W}+\mathrm{Rd}$ & 0.269 & 0.024 & 4.4 & 0.187 & 0.105 & 1377.8 \\
\hline $\mathrm{S}+\mathrm{LC}+\mathrm{Rd}$ & 0.265 & 0.030 & 5.0 & 0.180 & 0.120 & 1369.0 \\
\hline $\mathrm{S}+\mathrm{Rd}$ & 0.259 & 0.047 & 5.8 & 0.114 & 0.258 & 1304.6 \\
\hline $\mathrm{W}+\mathrm{Rd}$ & 0.240 & 0.026 & 8.0 & 0.198 & 0.067 & 424.0 \\
\hline $\mathrm{S}+\mathrm{LC}+\mathrm{W}+\mathrm{Riv}+\mathrm{Rd}$ & 0.239 & 0.045 & 8.2 & 0.182 & 0.115 & 1405.6 \\
\hline $\mathrm{S}+\mathrm{W}+\mathrm{Riv}+\mathrm{Rd}$ & 0.239 & 0.062 & 8.2 & 0.126 & 0.221 & 1272.6 \\
\hline $\mathrm{S}+\mathrm{Riv}+\mathrm{Rd}$ & 0.226 & 0.081 & 9.7 & 0.075 & 0.325 & 1274.9 \\
\hline
\end{tabular}

for frog species than salamanders (Smith \& Green 2005), a pattern that holds for R. sylvatica and A. maculatum (Berven \& Grudzien 1990; Semlitsch \& Skelly 2008). In addition, both $A$. maculatum and $R$. sylvatica exhibit high site fidelity to their natal pond, with slightly higher observed rates of philopatry for A. maculatum (Berven \& Grudzien 1990; Vasconcelos \& Calhoun 2004; Meier 2007). Shorter movement distances and higher site fidelity should lead to reduced gene flow and stronger differentiation among populations, which is consistent with the patterns I observed in A. maculatum relative to $R$. sylvatica.

Effective population size $\left(N_{\mathrm{e}}\right)$ and generation time differences may serve to countervail the influence of vagility and site fidelity in this system. A large $N_{\mathrm{e}}$ contains a correspondingly large reservoir of genetic variation, leading to a reduced influence of drift and slower rates of genetic change (Gauffre et al. 2008). Estimates from this study indicate that mean $N_{\mathrm{e}}$ is higher for A. maculatum than $R$. sylvatica, but the difference is not significant. Spotted salamanders are also longer lived than wood frogs (mean age $=7$ vs. 2.5 years), leading to increased iteroparity and overlapping generations in A. maculatum. This also can slow drift and genetic divergence by creating a temporal genetic reservoir, effectively increasing $N_{\mathrm{e}}$ (Nunney 1993). However, A. maculatum populations in this study exhibit greater differentiation than $R$. sylvatica despite higher $N_{e}$ estimates and overlapping generations.

In addition to vagility and generation time, other aspects of life history can influence genetic patterns. During each breeding cycle, A. maculatum individuals produce fewer offspring ( 200 eggs per female; Petranka 1998) than $R$. sylvatica females (mean of 774 eggs per female in this region; J.L. Richardson unpubl. data). A. maculatum also require a longer aquatic larval stage, with metamorphosis occurring an average of 5 weeks later than co-occurring $R$. sylvatica in this region (Paton \& Crouch 2002; Timm et al. 2007). Considering that 5 weeks is often the difference between a pond with water and a dry basin, the extra time required by A. maculatum may lead to lower rates of recruitment of aquatic larvae into terrestrial juveniles relative to $R$. sylvatica breeding in the same ponds (Petranka et al. 
2003; Lee 2008). For individuals of A. maculatum that do disperse, fewer offspring produced and a lower probability of recruitment may further hinder migrants and decrease gene flow relative to wood frogs. However, this effect would be buffered in part by the longer lifespan and iteroparity exhibited by salamanders. The 'storage effect' resulting from increased longevity and iteroparity has been found to be important for the long-term persistence of a closely related salamander (A. opacum; Taylor et al. 2006).

\section{Bayesian estimates of genetic structure}

Bayesian clustering highlighted differences in the genetic patterns exhibited by $A$. maculatum and $R$. sylvatica across the study region. In addition to different numbers of clusters being supported for each species, there was very little geographic overlap of genetic groups between species in the southern and middle part of the study region (Fig. 3). There was, however, geographic overlap in the most distinct cluster for both species, corresponding to the northern section of the Metacomet Ridge. This wood frog group expanded into three other populations and clearly encompassed all of the northern populations sampled (Fig. 3). There are no specific features that consistently coincide with the genetic breaks between clusters for either species, which may suggest that no particular landscape element acts as an absolute barrier to gene flow.

\section{Landscape features shaping genetic structure}

Results from resistance modelling indicate that different processes and landscape features influence the genetic patterns observed in each species. In salamanders, the best-supported models all included the river feature (Table 2; Table S6, Supporting information), while the geographic distance model (IBD) was ranked fifth using $F_{\mathrm{ST}}$ (third using $D_{\mathrm{ps}}$ ). For wood frogs, IBD was the bestsupported model, while the following nine most supported models (using $F_{\mathrm{ST}}$ ) all included the road feature. This suggests that both geographic distance and the presence of roads, particularly divided highways, shape the spatial genetic structure of wood frogs. This is in sharp contrast to the salamander analysis, where rivers were supported while the model with only roads ranked much lower (Table S6, Supporting information). Conversely, the river model was the least supported for the wood frog. The large discrepancy in model support provides strong evidence that these two species are experiencing different landscape-level effects on dispersal.

One biological interpretation of the support for different landscape features relates to the temporal presence of each feature on the landscape. If A. maculatum inherently move less than R. sylvatica, the lower rates of dispersal should be less impacted by any feature over short time periods. High-traffic roads are a relatively recent addition to the landscape ( $\sim 60$ years), and the time elapsed may be insufficient to produce a signal of restricted gene flow in A. maculatum. On the other hand, the slower diffusion of $A$. maculatum genes across the landscape has had several thousand years to be impacted by rivers. It is possible that a similar effect of roads cannot be detected yet due to genetic marker limitations (i.e. lag time between landscape modification and marker response) in combination with several life history attributes of A. maculatum (Anderson et al. 2010; Landguth et al. 2010). However, given the lack of any effect of rivers on $R$. sylvatica, there is a clear signal of differential response between the species that does not suffer from prospective marker limitations. Interestingly, a field study of two ranid frog species in Canada found that populations of the more vagile species were more negatively impacted by roads and traffic intensity than the less vagile sister species (Carr \& Fahrig 2001). Additionally, differences in longevity may play an important role in the genetic patterns observed. R. sylvatica experiencing a shorter lifespan have had more generations elapsed since the creation of roads, which should allow a genetic signal to be detected sooner than in the longer-lived $A$. maculatum (Landguth et al. 2010). Alternatively, it is possible that the signal attributed to roads is detecting another variable that is correlated with roads, but not directly measured in this study (e.g. historical settlement patterns, underlying geology).

Results from previous studies suggest that spatial genetic patterns in pond-breeding amphibians are highly dependent upon the landscape being evaluated. Variable signals of IBD suggest that this process is not universal in R. sylvatica or A. maculatum (Newman \& Squire 2001; Zamudio \& Wieczorek 2007; Crosby et al. 2009; Purrenhage et al. 2009). In R. sylvatica, estimates of genetic differentiation are consistently low $\left(F_{\mathrm{ST}}=0.01-0.025\right)$, and across a large region Lee-Yaw et al. (2009) identified the ocean and large lakes as the only predictors of genetic structure. Zellmer \& Knowles (2009) found evidence that slope, rivers and lakes reduce connectivity among $R$. sylvatica populations in Michigan. Consistent with the current study, other researchers have reported higher genetic differentiation in A. maculatum $\left(F_{\mathrm{ST}}=0.04-0.07\right) . F_{\mathrm{ST}}$ and slope were strongly correlated for A. maculatum in New York (Greenwald et al. 2009), while Ohio populations showed no association between genetic distance, slope or land cover (Purrenhage et al. 2009). Considering the extensive overlap in microsatellite loci used, the lack of commonalities among studies of these species (aside 
from higher $F_{\mathrm{ST}}$ in $A$. maculatum) could arise due to differences in the spatial scales investigated, distinct analytical approaches or landscape-specific processes affecting each region (Short Bull et al. 2011). In related species inhabiting more topographically complex landscapes, there are much stronger patterns of genetic structure commonly associated with elevation and topography (Tallmon et al. 2000; Funk et al. 2005; Spear et al. 2005; Giordano et al. 2007; Savage et al. 2010).

\section{Multiple species comparisons}

Studies evaluating multiple species within a landscape have found several similarities and important differences in spatial genetic patterns. Delaney et al. (2010) found similar gene flow reductions in response to urban development for three lizards and one bird species. Among three species of Pacific salmon, broad similarities in genetic patterns were observed relating to precipitation (Olsen et al. 2011). At smaller scales, however, genetic divergence and watershed effects differed among the species. Brede \& Beebee (2004) observed distinct levels of genetic differentiation for a toad (B. bufo) and frog species (R. temporaria) in England. In a study of two stream-breeding salamanders, Steele et al. (2009) found substantial differences in the genetic structure of the two species. These differences were linked to each species' dispersal ability, consistent with the patterns identified in the current study. Goldberg \& Waits (2010a) investigated landscape effects on two amphibian species closely related to A. maculatum and $R$. sylvatica in Idaho, USA. They found higher levels of genetic differentiation in both species relative to the current study; however, the focal species did not differ significantly from each other. Genetic distance was associated with land cover for both species, but the relative permeability of each cover type was different between species; long-toed salamander connectivity was highest in forested areas expected to support higher soil moisture, while gene flow among Columbia spotted frog populations was highest in agriculture and other areas thought to be low moisture environments. The differences between species are consistent with the current study, however, the effect of each land cover class did not differ substantially for spotted salamanders and wood frogs in this study. Collectively, studies of multiple species suggest that there are few obvious generalities, with several studies finding concordant landscape genetic responses among species while others have not. It does seem clear from past studies that the landscape context being evaluated has a strong influence on the genetic patterns observed - perhaps an intuitive outcome in landscape genetics.

\section{Evaluating multiple dispersal pathways}

Commonly, studies modelling the influence of landscape features use least-cost path or straight-line connections between sampling sites. Least-cost path analyses can be particularly useful for investigating fine-scale landscape genetic processes when the dispersal route options are limited (Wang et al. 2009; Sawyer et al. 2011). Network or transect-based methods do not require a resistance surface with designated costs for features, thereby reducing subjectivity (Rayfield et al. 2011; Emaresi et al. 2011) and can be useful for assessing the role of between and within-site processes on genetic patterns (Murphy et al. 2010). However, both least-cost path and network approaches restrict movement to a single dispersal path, which is a less biologically realistic way to model movement at larger spatial scales where gene flow occurs over a wider area and across multiple generations (McRae \& Beier 2007; Sawyer et al. 2011). The present study included many populations separated by distances that would be impossible for individual animals to traverse. In this context, modelling single dispersal routes would be unrealistic and of limited value. The circuit-based approach used in this study was able to simultaneously evaluate all dispersal pathways across the landscape, providing the most appropriate method to model the gene flow of amphibians across this region (McRae \& Beier 2007).

To select resistances for each landscape feature, I adopted the approach of Pérez-Espona et al. (2008) and tested a range of cost values for each feature against the genetic data. This approach avoids some of the subjectivity associated with assigning costs based on expert opinion. However, using genetic data to both establish costs and as a response variable to test landscape models is not ideal. Advanced methods will need to be developed to avoid some of the issues associated with resistance surface parameterization (Rayfield et al. 2010; Spear et al. 2010). As computational resources increase, landscape genetic analyses will benefit from multiparameter exploration, where resistance costs are optimized concurrently based on the association with and behaviour of other resistance features and their costs. Multiple regression, path analysis or structural equation modelling may provide a suitable framework, but currently, this approach is probably not feasible for large areas or with many landscape features in a program like CIRCUITSCAPE.

\section{Conclusions}

Simultaneously investigating two ecologically similar species using a variety of approaches illuminated important differences in the response of each species to the 
landscape. Additionally, the differences observed can be linked to the dispersal and life history attributes of each species. The results of this study are at odds with a presumption that landscape features have comparable impacts on taxonomically similar species. This is especially true of pond-breeding amphibians that are often classified as poor dispersers and are tied closely to water and their natal ponds. Whether it is a matter of our perception of landscape impediments or related to the practical limitations of data collection, we often treat specific landscape features as having homogenous impacts on species. However, when the stated goal of many studies is to provide information useful for conservation efforts, insights from multiple species are more valuable for developing comprehensive management strategies (Roberge \& Angelstam 2004). Unfortunately, evidence that landscape influence on population connectivity differs between species does not make the task of designing these strategies any easier. However, appreciating these complexities should help avoid some of the pitfalls associated with single-species management approaches, with the added benefit of advancing our understanding of movement more generally across species.

\section{Acknowledgements}

I thank D. Skelly, K. Zamudio and A. Caccone for helpful discussions throughout the course of this project. I thank R. Garrick, C. Goldberg, Q. Hyseni, and I. Wang for fruitful conversations on study design, theoretical underpinnings and suitable analyses, as well as C. Mariani and B. Evans for facilitating my lab work at the Yale Molecular Systematics and Conservation Genetics Center. D. Tomlin and E. Fournier provided valuable guidance on GIS methods; R. Waples provided helpful advice on $\mathrm{N}_{\mathrm{e}}$ estimation; B. McRae provided important insights related to CIRCUITSCAPE; and A. Zellmer graciously shared primer information. R. Garrick, D. Hawlena, W. Lowe, S. Spear, M. Urban and three anonymous reviewers provided valuable feedback on this manuscript. This research was supported by grants from the National Fish and Wildlife Foundation and National Geographic Society, and by an NSF graduate research fellowship.

\section{References}

Allendorf FW, Luikart G (2007) Conservation and the Genetics of Populations. Blackwell, Malden, Massachusetts.

Anderson CD, Epperson BK, Fortin MJ et al. (2010) Considering spatial and temporal scale in landscapegenetic studies of gene flow. Molecular Ecology, 19, 35653575.

Balkenhol N, Waits LP, Dezzani RJ (2009) Statistical approaches in landscape genetics: an evaluation of methods for linking landscape and genetic data. Ecography, 32, 818-830.

Berven KA, Grudzien TA (1990) Dispersal in the wood frog (Rana sylvatica) - implications for genetic population structure. Evolution, 44, 2047-2056.
Boone RB, Johnson CM, Johnson LB (2006) Simulating wood frog movement in central Minnesota, USA using a diffusion model. Ecological Modelling, 198, 255-262.

Bowcock AM, Ruiz-Linares A, Tomfohrde J, Minch E, Kidd JR, Cavalli-Sforza LL (1994) High resolution of human evolutionary trees with polymorphic microsatellites. Nature, 368, 455-457.

Brede EG, Beebee TJC (2004) Contrasting population structures in two sympatric anurans: implications for species conservation. Heredity, 92, 110-117.

Burnham KP, Anderson DR (2002) Model Selection and Multimodel Inference: A Practical Information-Theoretic Approach. Springer, New York, New York.

Canty A, Ripley B (2011) boot: Bootstap R Functions. $R$ package version 1.3. http:/ / www.cran.r-project.org/ package=boot.

Carr LW, Fahrig L (2001) Effect of road traffic on two amphibian species of differing vagility. Conservation Biology, 15, 1071-1078.

Castellano S, Balletto E (2002) Is the partial Mantel test inadequate? Evolution, 56, 1871-1873.

Chan LM, Zamudio KR (2009) Population differentiation of temperate amphibians in unpredictable environments. Molecular Ecology, 18, 3185-3200.

Chapuis M-P, Estoup A (2007) Microsatellite null alleles and estimation of population differentiation. Molecular Biology and Evolution, 24, 621-631.

Crosby MKA, Licht LE, Fu J (2009) The effect of habitat fragmentation on finescale population structure of wood frogs (Rana sylvatica). Conservation Genetics, 10, 1707-1718.

Crow JF, Kimura M (1970) An Introduction to Population Genetics Theory. Harper and Row, New York, New York.

Cunningham JM, Calhoun AJK, Glanz WE (2007) Pondbreeding amphibian species richness and habitat selection in a beaver-modified landscape. Journal of Wildlife Management, 71, 2517-2526.

Delaney KS, Riley SPD, Fisher RN (2010) A rapid, strong, and convergent genetic response to urban habitat fragmentation in four divergent and widespread vertebrates. PLOS ONE, 5, e12767.

Emaresi G, Pellet J, Dubey S, Hirzel AH, Fumagalli L (2011) Landscape genetics of the Alpine newt (Mesotriton alpestris) inferred from a strip-based approach. Conservation Genetics, 12, 41-50.

Evanno G, Regnaut S, Goudet J (2005) Detecting the number of clusters of individuals using the software STRUCTURE: a simulation study. Molecular Ecology, 14, 2611-2620.

Flageole S, Leclair R (1992) Demography of a salamander (Ambystoma maculatum) population studied by skeletochronology. Canadian Journal of Zoology, 70, 740-749.

Funk WC, Blouin MS, Corn PS et al. (2005) Population structure of Columbia spotted frogs (Rana luteiventris) is strongly affected by the landscape. Molecular Ecology, 14, 483-496.

Gauffre B, Estoup A, Bretagnolle V, Cosson J (2008) Spatial genetic structure of a small rodent in a heterogeneous landscape. Molecular Ecology, 17, 4619-4629.

Giordano AR, Ridenhour BJ, Storfer A (2007) The influence of altitude and topography on genetic structure in the longtoed salamander (Ambystoma macrodactulym). Molecular Ecology, 16, 1625-1637. 
Goldberg CS, Waits LP (2010a) Comparative landscape genetics of two pond-breeding amphibian species in a highly modified agricultural landscape. Molecular Ecology, 19, 36503663.

Goldberg CS, Waits LP (2010b) Quantification and reduction of bias from sampling larvae to infer population and landscape genetic structure. Molecular Ecology Resources, 10, 304-313.

Goudet J (1995) FSTAT (version 1.2): a computer program to calculate F-statistics. Journal of Heredity, 86, 485-486.

Greenwald KR, Purrenhage JL, Savage WK (2009) Landcover predicts isolation in Ambystoma salamanders across region and species. Biological Conservation, 142, 2493-2500.

Guillot G, Leblois R, Coulon A, Frantz AC (2009) Statistical methods in spatial genetics. Molecular Ecology, 18, 4734-4756.

Halverson MA, Skelly DK, Caccone A (2006) Kin distribution of amphibian larvae in the wild. Molecular Ecology, 15, 11391145.

Hedrick PW (2005) A standardized genetic differentiation measure. Evolution, 59, 1633-1638.

Holm S (1979) A simple sequentially rejective multiple test procedure. Scandinavian Journal of Statistics, 6, 65-70.

Hothorn T, Hornik K (2006) exactRankTests: exact Distributions for Rank and Permutation Tests. $R$ package version 0.8-17. http://www.cran.r-project.org/package=exact RankTests.

Hubisz MJ, Falush D, Stephens M, Pritchard JK (2009) Inferring weak population structure with the assistance of sample group information. Molecular Ecology Resources, 9, 1322-1332.

Jakobsson M, Rosenberg NA (2007) CLUMPP: a cluster matching and permutation program for dealing with label switching and multimodality in analysis of population structure. Bioinformatics, 23, 1801-1806.

Jenkins DG, Carey M, Czerniewska J et al. (2010) A metaanalysis of isolation by distance: relic or reference standard for landscape genetics? Ecography, 33, 315-320.

Jost L (2008) $G_{\mathrm{ST}}$ and its relatives do not measure differentiation. Molecular Ecology, 17, 4015-4026.

Landguth EL, Cushman SA, Schwartz MK, McKelvey KS, Murphy M, Luikart G (2010) Quantifying the lag time to detect barriers in landscape genetics. Molecular Ecology, 19, 4179-4191.

Lee C (1985) West Rock to the Barndoor Hills, The Traprock Ridges of Connecticut. CT DEP, Hartford, Connecticut.

Lee EH (2008) Predicting Wood Frog Population Dynamics. PhD Thesis, Yale University, New Haven.

Lee-Yaw JA, Irwin JT, Green DM (2008) Postglacial range expansion from northern refugia by the wood frog, Rana sylvatica. Molecular Ecology, 17, 867-884.

Lee-Yaw JA, Davidson A, McRae BH, Green DM (2009) Do landscape processes predict phylogeographic patterns in the wood frog? Molecular Ecology, 18, 1863-1874.

Legendre P, Fortin MJ (2010) Comparison of the Mantel test and alternative approaches for detecting complex multivariate relationships in the spatial analysis of genetic data. Molecular Ecology Resources, 10, 831-844.

Lesbarrères D, Primmer CR, Lodé T, Merilä J (2006) The effects of 20 years of highway presence on the genetic structure of Rana dalmatina populations. Ecoscience, 13, 531-538.
Manel S, Schwartz MK, Luikart G, Taberlet P (2003) Landscape genetics: combining landscape ecology and population genetics. Trends in Ecology \& Evolution, 18, 189-197.

Manier MK, Arnold SJ (2006) Ecological correlates of population genetic structure: a comparative approach using a vertebrate metacommunity. Proceedings of the Royal Society $B, 273,3001-3009$.

McRae BH, Beier P (2007) Circuit theory predicts gene flow in plant and animal populations. Proceedings of the National Academy of Sciences, 104, 19885-19890.

McRae BH, Dickson BG, Keitt TH, Shah VB (2008) Using circuit theory to model connectivity in ecology, evolution, and conservation. Ecology, 89, 2712-2724.

Meier PT (2007) Fine spatial scale phenotypic divergence in wood frogs (Lithobates sylvaticus). Canadian Journal of Zoology, 85, 873-882.

Meirmans PG (2006) Using the AMOVA framework to estimate a standardized genetic differentiation measure. Evolution, 60, 2399-2402.

Meirmans PG, Hedrick PW (2011) Assessing population structure: $F_{\mathrm{ST}}$ and related measures. Molecular Ecology Resources, 11, 5-18.

Montieth KE, Paton PWC (2006) Emigration behavior of spotted salamanders on golf courses in southern Rhode Island. Journal of Herpetology, 40, 195-205.

Murphy MA, Dezzani R, Pilliod DS, Storfer A (2010) Landscape genetics of high mountain frog metapopulations. Molecular Ecology, 19, 3634-3649.

Newman RA, Squire T (2001) Microsatellite variation and finescale population structure in the wood frog (Rana sylvatica). Molecular Ecology, 10, 1087-1100.

Nicholson E, Possingham HP (2006) Objectives for multiplespecies conservation planning. Conservation Biology, 20, 871881.

Nunney L (1993) The influence of mating system and overlapping generations on effective population size. Evolution, 47, 1329-1341.

Olsen JB, Crane PA, Flannery BG, Dunmall K, Templin WD, Wenburg JK (2011) Comparative landscape genetic analysis of three Pacific salmon species from subarctic North America. Conservation Genetics, 12, 223-241.

Park S (2001) The Excel Microsatellite Toolkit (v3.1). Animal Genomics Laboratory, UCD, Ireland.

Paton PWC, Crouch WB (2002) Using the phenology of pondbreeding amphibians to develop conservation strategies. Conservation Biology, 16, 194-204.

Pérez-Espona S, Pérez-Barbería FJ, McLeod JE, Jiggins CD, Gordon IJ, Pemberton JM (2008) Landscape features affect gene flow of Scottish Highland red deer (Cervus elaphus). Molecular Ecology, 17, 981-996.

Petranka JW (1998) Salamanders of the United States and Canada. Smithsonian, Washington, District of Columbia.

Petranka JW, Murray SS, Kennedy CA (2003) Responses of amphibians to restoration of a southern Appalachian wetland: perturbations confound post-restoration assessment. Wetlands, 23, 278-290.

Pritchard JK, Stephens M, Donnelly P (2000) Inference of population structure using multilocus genotype data. Genetics, 155, 945-959.

Purrenhage JL, Niewiarowski PH, Moore FB-G (2009) Population structure of spotted salamanders (Ambystoma 
maculatum) in a fragmented landscape. Molecular Ecology, 18, 235-247.

R Development Core Team (2001) R: A Language and Environment for Statistical Computing. http:/ / www.r-project.org.

Raufaste N, Rousset F (2001) Are partial Mantel tests adequate? Evolution, 55, 1703-1705.

Rayfield B, Fortin MJ, Fall A (2010) The sensitivity of least-cost habitat graphs to relative cost surface values. Landscape Ecology, 25, 519-532.

Rayfield B, Fortin MJ, Fall A (2011) Connectivity for conservation: a framework to classify network measures. Ecology, 92, 847-858.

Roberge JM, Angelstam P (2004) Usefulness of the umbrella species concept as a conservation tool. Conservation Biology, 18, 76-85.

Rosenberg N (2004) DISTRUCT: a program for the graphical display of population structure. Molecular Ecology Notes, 4, 137-138.

Sagor ES, Ouellet M, Barten E, Green DM (1998) Skeletochronology and geographic variation in age structure in the wood frog Rana sylvatica. Journal of Herpetology, 32, 469-474.

Savage WK, Fremier AK, Shaffer HB (2010) Landscape genetics of alpine Sierra Nevada salamanders reveal extreme population subdivision in space and time. Molecular Ecology, 19, 3301-3314.

Sawyer SC, Epps CW, Brashares JS (2011) Placing linkages among fragmented habitats: do least-cost models reflect how animals use landscapes? Journal of Applied Ecology, 48, 668678 .

Schwenk W, Donovan T (2011) A multispecies framework for landscape conservation planning. Conservation Biology, 25, 1010-1021.

Scribner KT, Arntzen JW, Cruddace N, Oldham RS, Burke T (2001) Environmental correlates of toad abundance and population genetic diversity. Biological Conservation, 98, 201210.

Semlitsch RD, Skelly DK (2008) Ecology and conservation of pool-breeding amphibians. In: Science and Conservation of Vernal Pools in Northeastern North America (eds Calhoun AJK and deMaynadier PG), pp. 127-147. CRC Press, Boca Raton, Florida.

Short Bull RA, Cushman SA, Mace R et al. (2011) Why replication is important in landscape genetics: American black bear in the Rocky Mountains. Molecular Ecology, 20, 1092-1107.

Smith MA, Green DM (2005) Dispersal and the metapopulation paradigm in amphibian ecology and conservation: are all amphibian populations metapopulations? Ecography, 28, 110128

Spear SF, Peterson CR, Matocq MD, Storfer A (2005) Landscape genetics of the blotched tiger salamander (Ambystoma tigrinum melanostictum). Molecular Ecology, 14, 2553-2564

Spear SF, Balkenhol N, Fortin MJ, McRae BH, Scribner K (2010) Use of resistance surfaces for landscape genetic studies: considerations for parameterization and analysis. Molecular Ecology, 19, 3576-3591.

Steele CA, Baumsteiger J, Storfer A (2009) Influence of lifehistory variation on the genetic structure of two sympatric salamander taxa. Molecular Ecology, 18, 1629-1639.
Storfer A, Murphy MA, Evans JS et al. (2007) Putting the 'landscape' in landscape genetics. Heredity, 98, 128-142.

Tallmon DA, Funk WC, Dunlap WW, Allendorf FW (2000) Genetic differentiation among Long-Toed Salamander (Ambystoma macrodactylum) populations. Copeia, 2000, 27-35.

Taylor BE, Scott DE, Gibbons JW (2006) Catastrophic reproductive failure, terrestrial survival, and persistence of the marbled salamander. Conservation Biology, 20, 792-801.

Timm BC, McGarigal K, Gamble LR (2007) Emigration timing of juvenile pond-breeding amphibians in Western Massachusetts. Journal of Herpetology, 41, 243-250.

Van Oosterhout C, Hutchinson WF, Wills DPM, Shipley P (2004) MICRO-CHECKER: software for identifying and correcting genotyping errors in microsatellite data. Molecular Ecology Notes, 4, 535-538.

Vasconcelos D, Calhoun AJK (2004) Movement patterns of adult and juvenile Rana sylvatica and Ambystoma maculatum in three restored seasonal pools in Maine. Journal of Herpetology, 38, 551-561.

Wang J (2004) Sibship reconstruction from genetic data with typing errors. Genetics, 166, 1963-1979.

Wang IJ, Savage WK, Shaffer HB (2009) Landscape genetics and least-cost path analysis reveal unexpected dispersal routes in the California tiger salamander (Ambystoma californiense). Molecular Ecology, 18, 1365-1374.

Waples RS, Do C (2008) LDNE: a program for estimating effective population size from data on linkage disequilibrium. Molecular Ecology Resources, 8, 753-756.

Weir BS, Cockerham CC (1984) Estimating F-statistics for the analysis of population structure. Evolution, 38, 1358-1370.

Whitlock M (2011) $G_{\mathrm{ST}}^{\prime}$ and $D$ do not replace $F_{\mathrm{ST}}$. Molecular Ecology, 20, 1083-1091.

Zamudio KR, Savage WK (2003) Historical isolation, range expansion, and secondary contact of two highly divergent mitochondrial lineages in spotted salamanders (Ambystoma maculatum). Evolution, 57, 1631-1652.

Zamudio KR, Wieczorek AM (2007) Fine-scale spatial genetic structure and dispersal among spotted salamander (Ambystoma maculatum) breeding populations. Molecular Ecology, 16, 257-274.

Zellmer A, Knowles LL (2009) Disentangling the effects of historic vs. contemporary landscape structure on population genetic divergence. Molecular Ecology, 18, 3593-3602.

J.L.R. is a PhD student using genetic assays and field experiments to examine how landscape structure affects wildlife movement, and how consequent levels of landscape connectivity influence population dynamics and evolution.

\section{Data accessibilty}

Microsatellite data for each species and population: DRYAD entry doi: $10.5061 /$ dryad.51b94

\section{Supporting information}

Additional supporting information may be found in a separate document included with this submission: 
Table S1 Sampling site information, including sample sizes, $\mathrm{N}_{\mathrm{e}}$ estimates and genetic summary statistics.

Table S2 Matrix of pairwise $F_{\mathrm{ST}}$ values for spotted salamanders (Ambystoma maculatum).

Table S3 Matrix of pairwise $F_{\mathrm{ST}}$ values for wood frogs (Rana sylvatica).

Table S4 Original land cover classifications from National Land Cover Database and the consolidated class assignments for the land cover landscape variable.

Table S5 Cost values evaluated for each landscape feature as part of Step 1 in the landscape resistant modelling component of this study.
Table S6 Full set of additive landscape resistance models assessed (continuation of Table 2).

Appendix S1 PCR conditions for primers used to amplify microsatellite loci.

Appendix S2 Expanded landscape resistance modelling methods.

Appendix S3 Expanded methods to test for nonindependence.

Please note: Wiley-Blackwell are not responsible for the content or functionality of any supporting information supplied by the authors. Any queries (other than missing material) should be directed to the corresponding author for the article. 